\title{
Solar Changes and the Climate
}

The sun plays a role in our climate in direct and indirect ways.

The sun changes in its activity on time scales that vary from 27 days to 11, 22, 80, 180 years and more. A more active sun is brighter due to the dominance of faculae over cooler sunspots with the result that the irradiance emitted by the sun and received by the earth is higher during active solar periods than during quiet solar periods. The amount of change of the solar irradiance based on satellite measurements since 1978 during the course of the 11 year cycle just $0.1 \%$ (Frohlich and Lean 1998) has caused many to conclude that the solar effect is negligible especially in recent years. Over the ultra long cycles (since the Maunder minimum), irradiance changes are estimated to be as high as 0.4\% (Hoyt and Schatten (1993), Lean et al. (1995), Lean (2000), Lockwood and Stamper (1999) and Fligge and Solanki (2000)).

However this does not take into account the sun's eruptional activity (flares, solar wind bursts from coronal mass ejections and solar wind bursts from coronal holes) which may have a much greater effect. This takes on more importance since Lockwood et al., 1999) showed how the total magnetic flux leaving the sun has increased by a factor of 2.3 since 1901. This eruptional activity may enhance warming through ultraviolet induced ozone chemical reactions in the high atmosphere or ionization in higher latitudes during solar induced geomagnetic storms. In addition, the work of Svensmark (1997), Bago and Butler (2000) Tinsley and Yu (2002) have documented the possible effects of the solar cycle on cosmic rays and through them the amount of low cloudiness. It may be that through these other indirect factors, solar variance is a much more important driver for climate change than currently assumed. Because, it is more easily measured and generally we find eruptional activity tracking well with the solar irradiance, we may utilize solar irradiance measurements as a surrogate or proxy for the total solar effect.

\section{Correlations with Total Solar Irradiance}

Studies vary on the importance of direct solar irradiance especially in recent decades. Lockwood and Stamper (GRL 1999), estimated that changes in solar luminosity can account for $52 \%$ of the change in temperatures from 1910 to 1960 but just $31 \%$ of the change from 1970 to 1999.

N Scafetta and B. J. West of Duke University, in "Phenomenological Solar Signature in 400 years of Reconstructed Northern Hemisphere Temperature Record” (GRL 2006) showed how total solar irradiance accounted for up to $50 \%$ of the warming since 1900 and $25-35 \%$ since 1980 . The authors noted the recent departures may result "from spurious non-climatic contamination of the surface observations such as heat-island and land-use effects [Pielke et al., 2002; Kalnay and Cai, 2003]”. There analysis was done using the global data bases which may also suffer from station dropout and improper adjustment for missing data which increased in the 1990s. 
This USHCN data base though regional in nature would have been a better station data base to use for analysis of change as it is more stable, has less missing data and a better scheme for adjusting for missing data, as well as some adjustments for changes to siting and urbanization. (Eliminated Pielke reference here)

An independent analysis was conducted using the USHCN data and TSI data obtained from Hoyt and Schatten. The annual TSI composite record was constructed by Hoyt and Schatten [1993] (and updated in 2005) utilizing all five historical proxies of solar irradiance including sunspot cycle amplitude, sunspot cycle length, solar equatorial rotation rate, fraction of penumbral spots, and decay rate of the 11-year sunspot cycle.

The following includes a plot of this latest 11-year running mean solar irradiance versus a similar 11-year running mean of NCDC annual mean US temperatures. It confirms this strong correlation (r-squared of 0.59 ). The correlation increases to an r-squared value of 0.654 if you introduce a lag of 3 years for the mean USHCN data to the mean TSI. This is close to the 5 year lag suggested by Wigley and used by Scafetta and West. The highest correlation occurred with a 3 year lag.

\section{NCDC Annual Mean US Temperature vs Hoyt Schatten TSI}

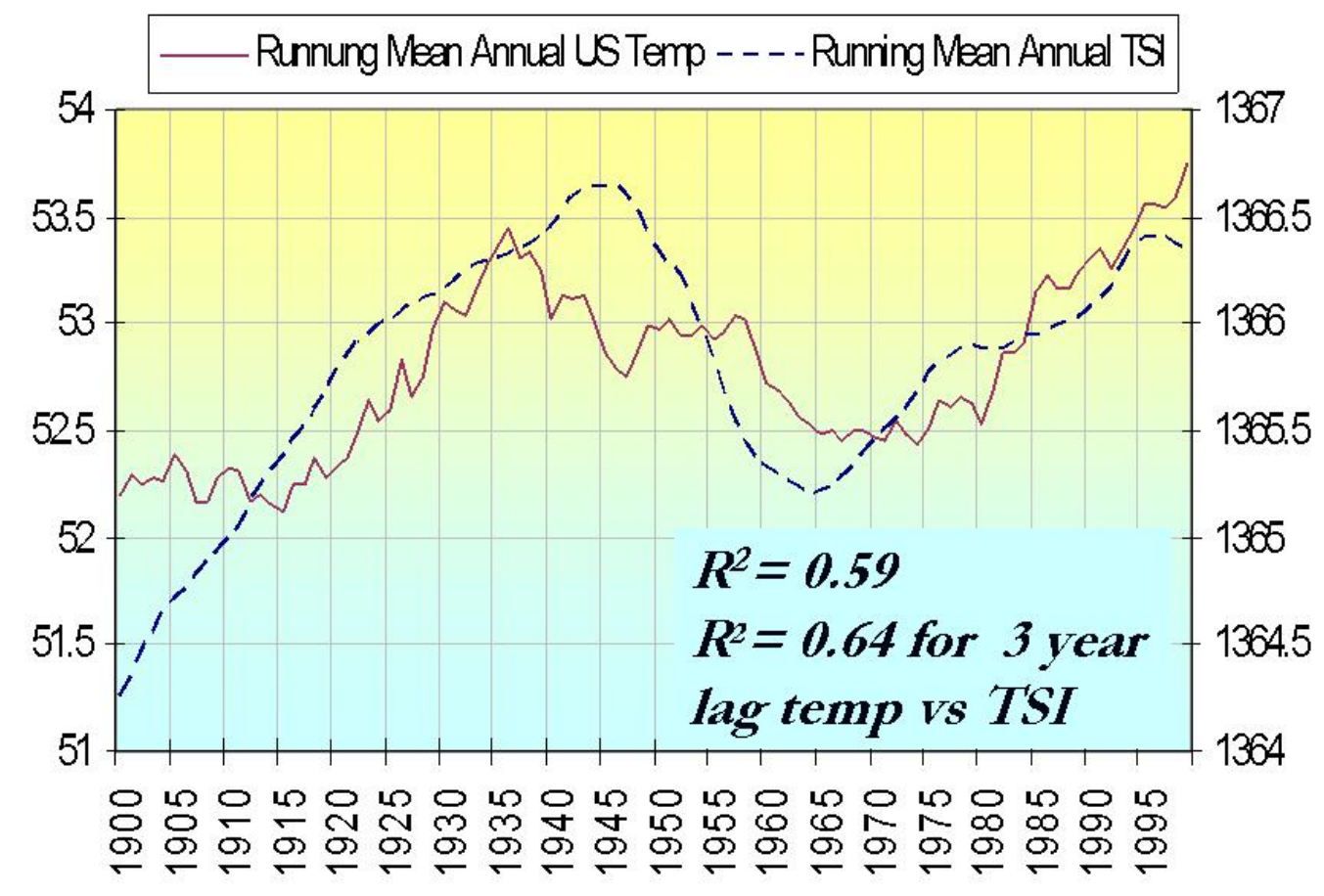

Gleissberg Cycle

Figure 1: USHCN Annual Mean Temperature (11 year running mean) correlated with Hoyt-Schatten Total Solar Irradiance (also 11 year running mean). 
In recent years, satellite missions designed to measure changes in solar irradiance though promising have produced there own set of problems. As Judith Lean noted the problems is that no one sensor collected data over the entire time period from 1979 "forcing a splicing of from different instruments, each with their own accuracy and reliability issues, only some of which we are able to account for”. Lean and Froelich in their 1998 GRL paper gave their assessment which suggested no increase in solar irradiance in the 1980s and 1990s.

Richard Willson, principal investigator of NASA's ACRIM experiments though in the GRL in 2003 was able to find specific errors in the dataset used by Lean and Froelich used to bridge the gap between the ACRIM satellites and when the more accurate data set was used a trend of $0.05 \%$ per decade was seen which could account for warming since 1979 (figure 2).

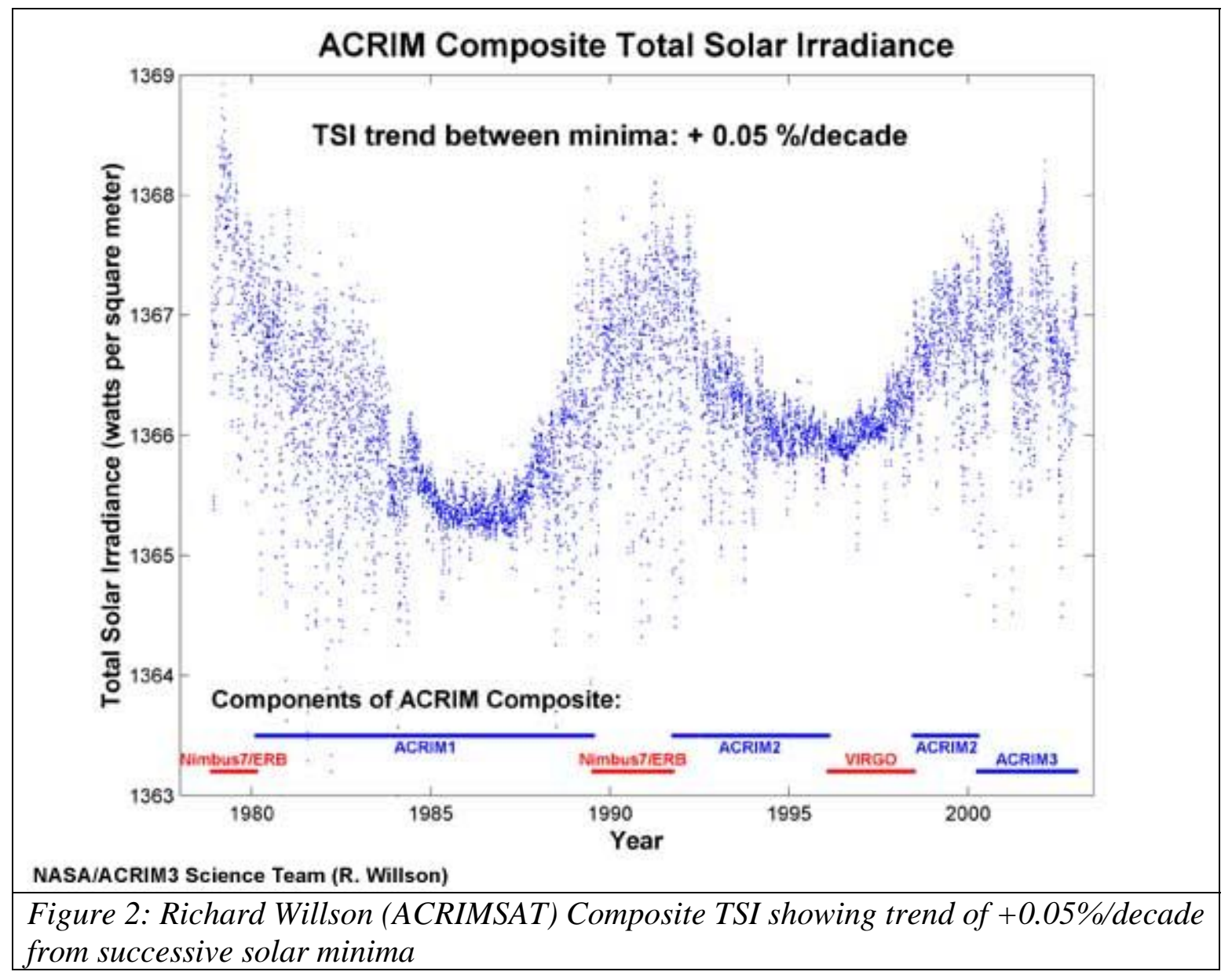

Two other recent studies that have drawn clear connections between solar changes and the Earth's climate are Soon (2005) and Kärner (2004). Soon (2005 GRL) showed how the arctic temperatures correlated with solar irradiance far better than with the greenhouse gases over the last century (see Figure 3). For the 10 year running mean of total solar irradiance (TSI) vs Arctic-wide air temperature anomalies (Polyokov), he found a strong 
correlation of (r-squared of 0.79) compared to a correlation vs greenhouse gases of just 0.22 .
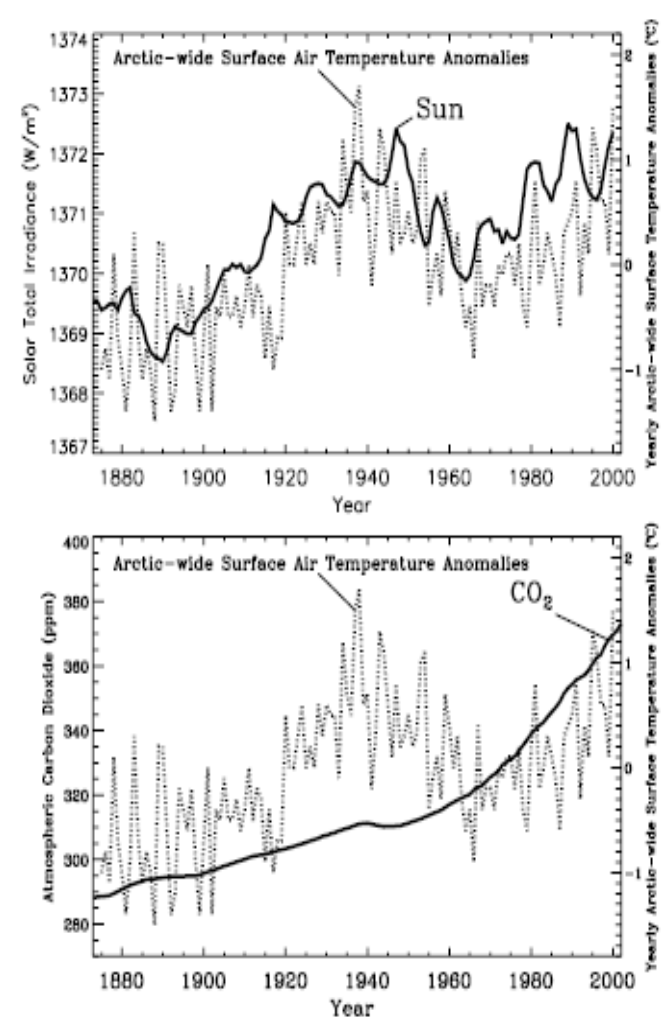

Figure 3. Arctic Basin wide air temperatures (Polyokov) correlated with Hoyt Schatten Total Solar Irradiance (TSI) and with annual average $\mathrm{CO}^{2}$ (Soon 2005)

Kärner (2002) studied the time series properties of daily total solar irradiance and daily average tropospheric and stratospheric temperature anomalies. He showed that average temperature anomalies exhibit a temporal evolution characterized by antipersistency, in which the variance expands as the observed sample length increases on all time scales, but at a diminishing total rate. $\mathrm{CO}_{2}$ forcing is not antipersistent, instead it has a steadily increasing trend, implying persistency. But he showed that total solar irradiance is antipersistent, implying a discriminating hypothesis: the dominant forcing mechanism will endow the atmospheric temperature data with its time series property. Since the temperature series is antipersistent this implies that solar forcing dominates. The test supported this finding on all available time scales, from daily to decadal. He concluded that:

"The revealed antipersistence in the lower tropospheric temperature increments does not support the science of global warming developed by IPCC [1996]. Negative long-range correlation of the increments during last 22 years means that negative feedback has been dominating in the Earth climate system during that period. The result is opposite to 
suggestion of Mitchell [1989] about domination of a positive cumulative feedback after a forced temperature change. Dominating negative feedback also shows that the period for $\mathrm{CO} 2$ induced climate change has not started during the last 22 years. Increasing concentration of greenhouse gases in the Earth atmosphere appeared to produce too weak forcing in order to dominate in the Earth climate system.” (Kärner 2002)

\section{Warming Due To Ultraviolet Effects Through Ozone Chemistry}

Though solar irradiance varies slightly over the 11 year cycle, radiation at longer UV wavelengths are known to increase by several percent with still larger changes (factor of two or more) at extremely short UV and X-ray wavelengths (Baldwin and Dunkerton, JAS 2004).

Energetic flares increase the UV radiation by $16 \%$. Ozone in the stratosphere absorbs this excess energy and this heat has been shown to propagate downward and affect the general circulation in the troposphere. Shindell (1999) used a climate model that included ozone chemistry to reproduce this warming during high flux (high UV) years. Labitzke and Van Loon (1988) and later Labitzke in numerous papers has shown that high flux (which correlates very well with UV) produces a warming in low and middle latitudes in winter in the stratosphere with subsequent dynamical and radiative coupling to the troposphere. The winter of 2001/02, when cycle 23 had a very strong high flux second maxima provided a perfect verification of Shindell and Labitzke and Van Loon's work. 


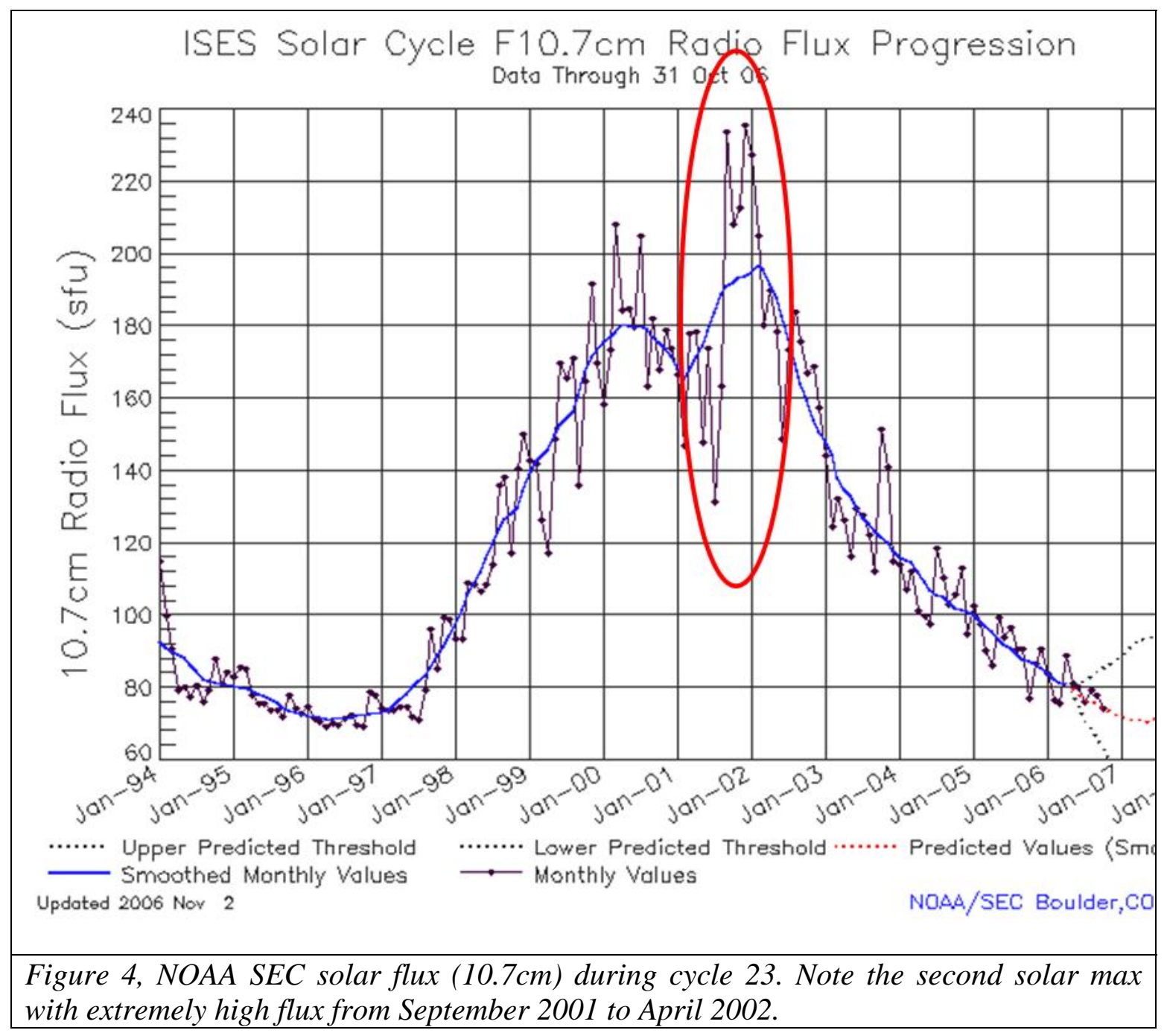




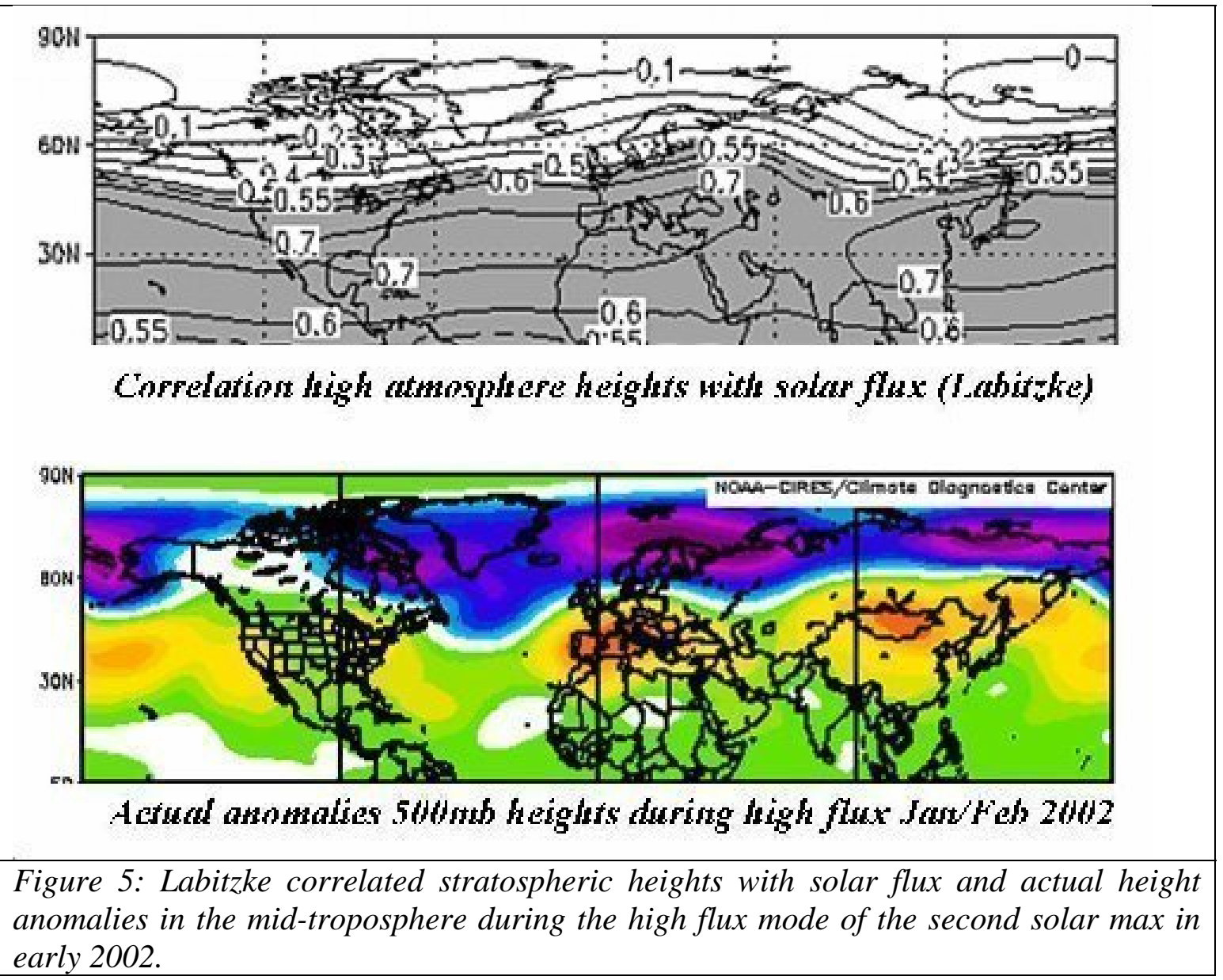

The warming that took place with the high flux from September 2001 to April 2002 caused the northern winter polar vortex to shrink and the southern summer vortex to break into two centers for the first time ever observed. This disrupted the flow patterns and may have contributed to the brief summer breakup of the Larsen ice sheet. 


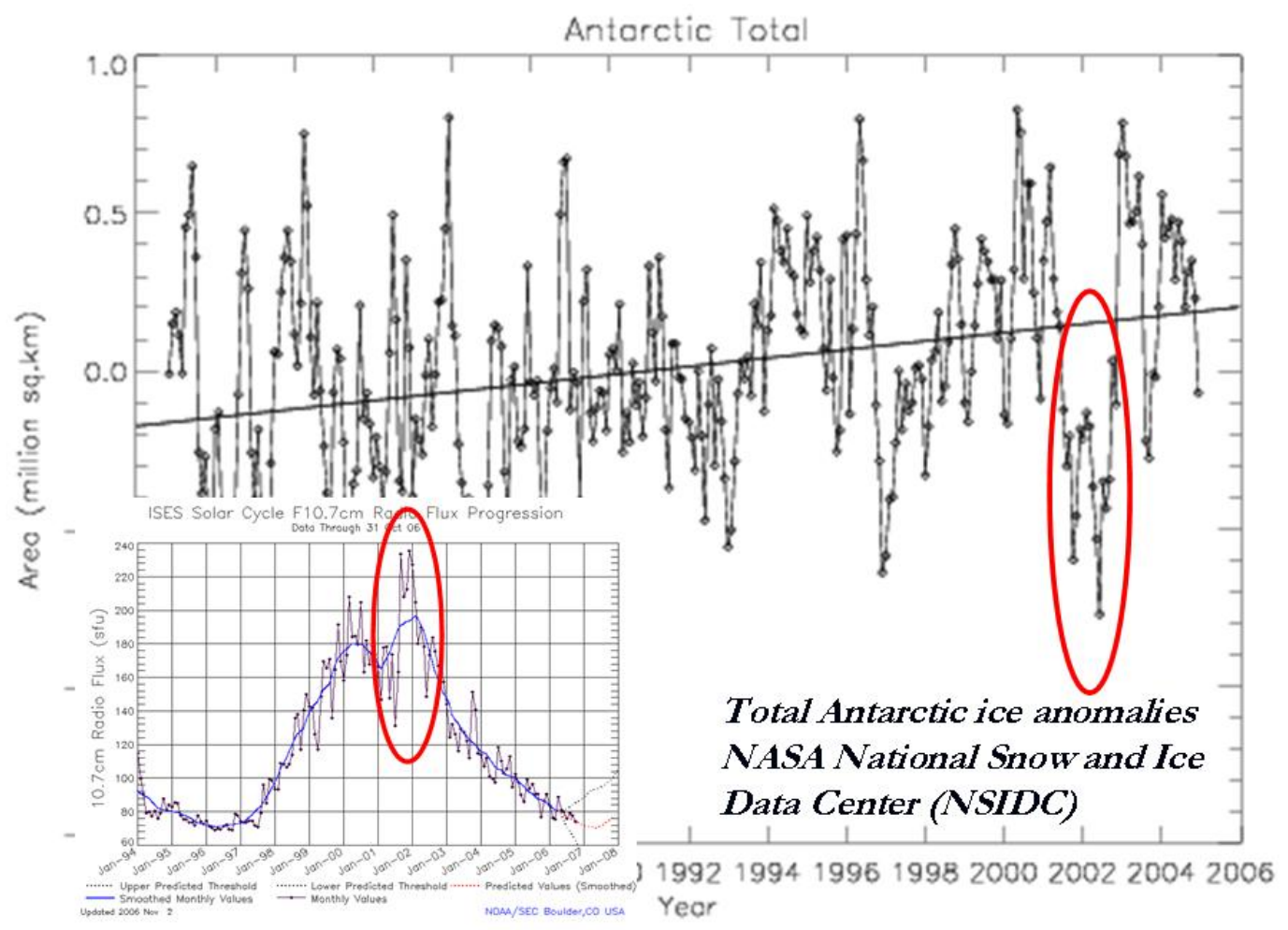

Figure 6: NASA NSIDC satellite derived Total Antarctic ice extent anomalies from 1979 to 2005. Note the dropoff with the Larsen ice sheet break-up in the summer of 2002 corresponding to major atmospheric changes during the high flux second solar max.

\section{Geomagnetic Activity and Global Temperatures}

When Gleissberg (1958) first investigated solar cycle lengths, he found short cycles had high sunspot numbers and strong eruptive activity while long cycles were characterized by low maxima and fewer solar eruptions. This may explain why Friis-Christensen and Lassen (1991) found a correlation of solar cycle length with temperatures. With major geomagnetic storms, there is ionization warming in the polar auroral zone, an increase in solar wind with more general warming. Landscheidt (2003) showed a r-squared correlation of 0.92 between smoothed yearly global temperature anomalies and a smoothed geomagnetic index (aa index) with a tendency for temperatures to lag the solar by 4 to 8 years. 


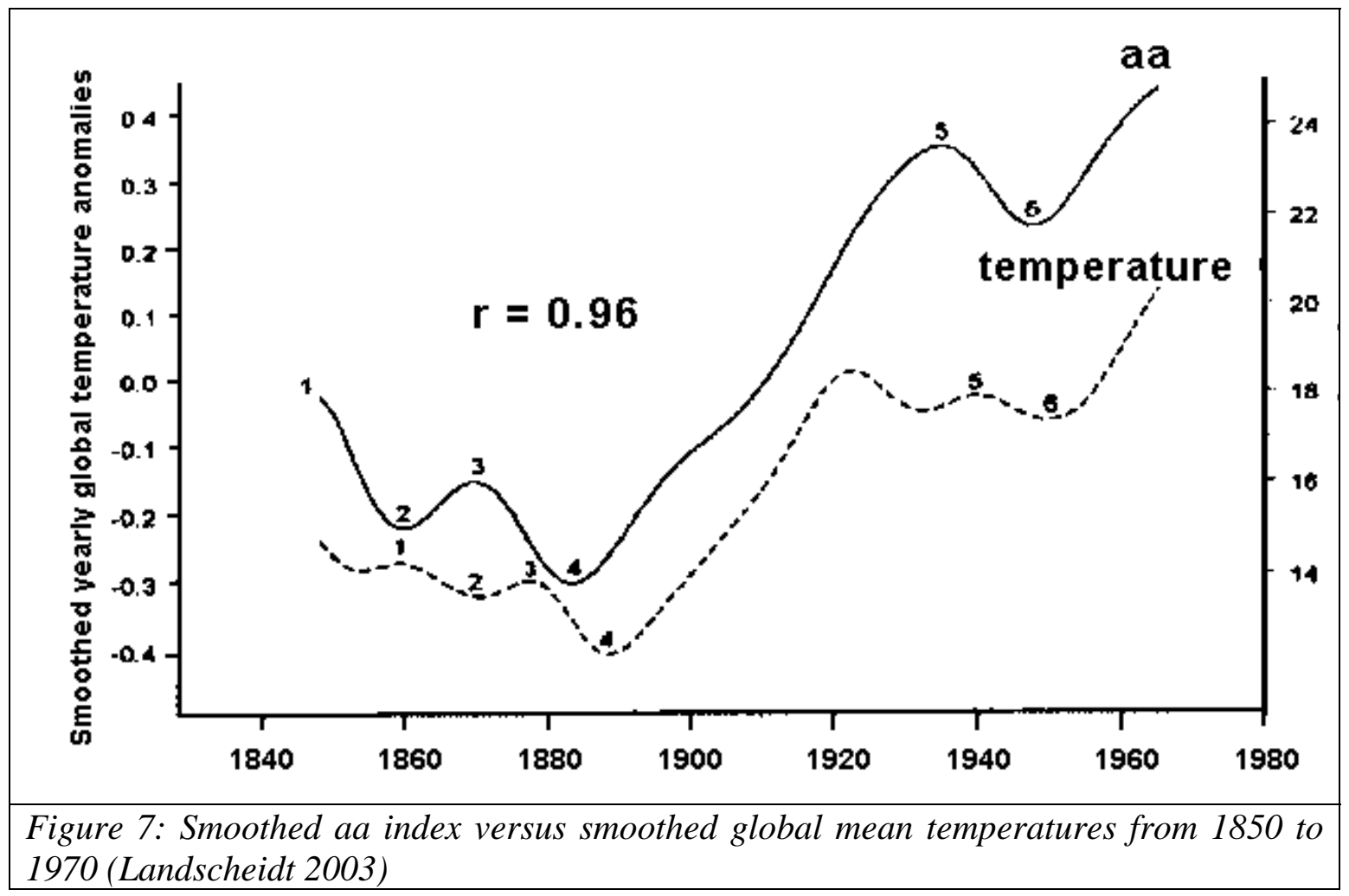

\section{Solar Winds, Cosmic Rays and Clouds}

A key aspect of the sun's effect on climate is the indirect effect on the flux of Galactic Cosmic Rays (GCR) into the atmosphere. GCR is an ionizing radiation that supports low cloud formation. As the sun's output increases the solar wind shields the atmosphere from GCR flux. Consequently the increased solar irradiance is accompanied by reduced low cloud cover, amplifying the climatic effect. Likewise when solar output declines, increased GCR flux enters the atmosphere, increasing low cloudiness and adding to the cooling effect associated with the diminished solar energy.

The conjectured mechanism connecting GCR flux to cloud formation received experimental confirmation in the recent laboratory experiments of Svensmark (Proceedings of the Royal Society, Series A, October 2006), in which he demonstrated exactly how cosmic rays could make water droplet clouds.

Palle Bago and Butler showed in 2002 (Intl J Climat.) how the low clouds in all global regions changed with the 11 year cycle in inverse relation to the solar activity. Changes of 1 to $2 \%$ in low cloudiness could have a significant effect on temperatures through chnges in albedo. 


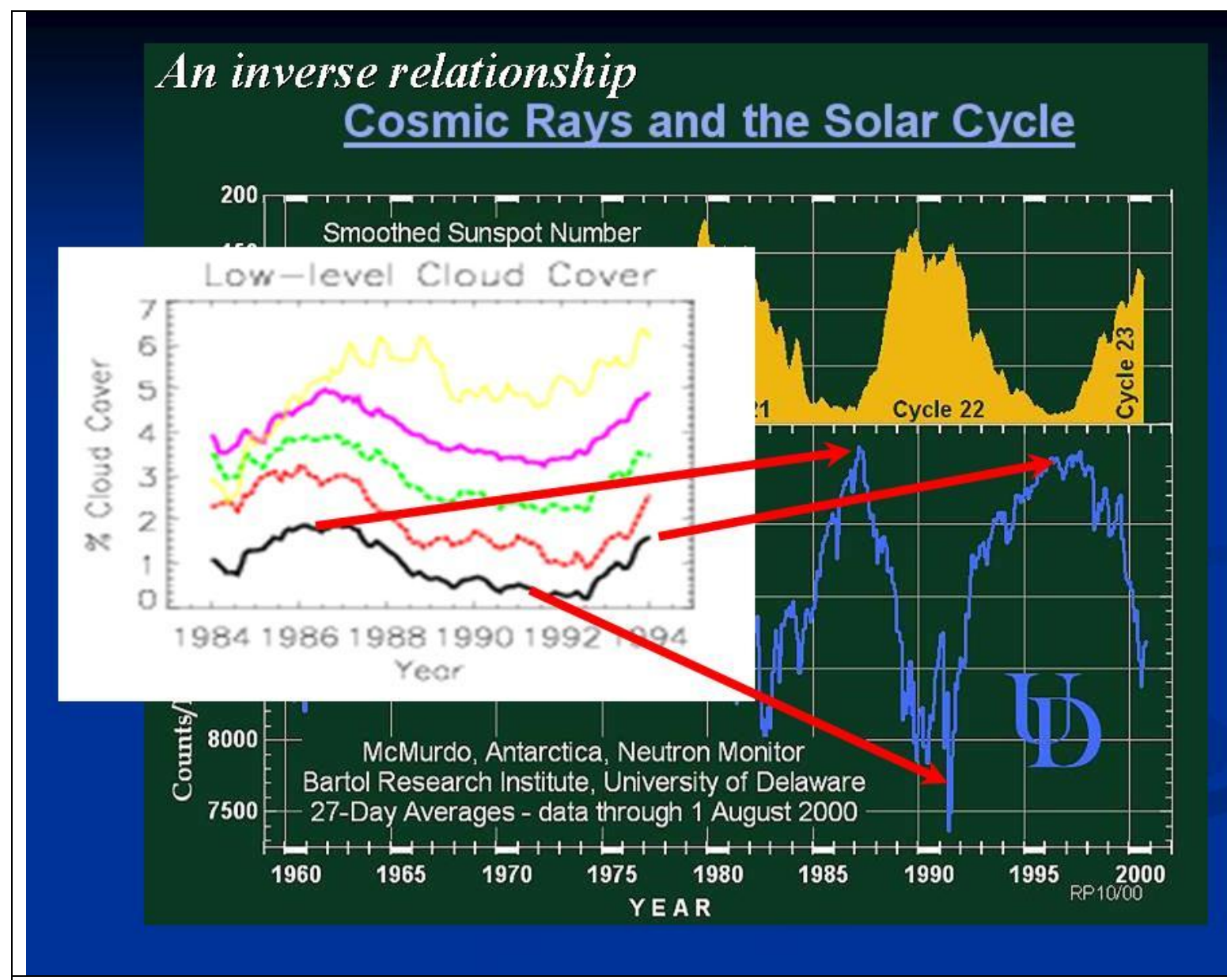

Figure 8: Cosmic ray neutrons are inversely proportional to solar activity and directly proportional to low cloudiness (cloud data from Palle-Bago and Butler 2002)

Shaviv (JGR 2005 estimated from the combination of increased radiative forcing through cosmic ray reduction and the estimated changes in total solar luminosity (irradiance) over the last century that the sun could be responsible for up to $77 \%$ of the temperature changes over the $20^{\text {th }}$ century with $23 \%$ for the anthropogenic.

Summary:

Though the sun's brightness or irradiance changes only slightly with the solar cycles, the indirect effects of enhanced solar activity including warming of the atmosphere in low and mid latitudes by ozone reactions due to increased ultraviolet radiation, in higher latitudes by geomagnetic activity and generally by increased radiative forcing due to less clouds caused by cosmic ray reduction may greatly magnify the total solar effect on temperatures.

\section{Solar Experts:}

Dr. Sallie Baliunas

Harvard-Smithsonian Center for Astrophysics 
60 Garden St.

Cambridge MA 02138

baliunas@cfa.harvard.edu

Dr. Richard C. Willson

Principal Investigator, ACRIM Experiments

Columbia University

12 Bahama Bend

Coronado, CA, 92118

Phone: 619-407-7716

rwillson@acrim.com

\section{References:}

Baldwin, M.P., Dunkeryton, T.J.: (2004) The solar cycle and stratospheric-tropsospheric dynamical coupling, JAS 2004

Baliunas, S., "Uncertainties in Climate Modeling: Solar Variability and Other Factors," September 17, 1996

Baliunas, S., Soon, W. "The Sun Also Warms", March 24, 2000

Baliunas, S., "Hot Times or Hot Air: The Sun in the Science of Global Warming," August 7, 1998

Baliunas, S., and Soon, $W .$, An Assessment of the Sun-Climate Relation on Time Scales of Decades to Centuries: the Possibility of Total Irradiance Variations Harvard Smithsonian Center for Astrophysics, 60 Garden Street, Cambridge, MA 02138, Mount Wilson Observatory, Mount Wilson, CA 91023

Baliunas, Sallie, "Uncertainties in Climate Modeling: Solar Variability and Other Factors," September 17, 1996

Boberg, F. and H. Lundstedt, 2002: Solar wind variations related to fluctuations of the North Atlantic Oscillation, Geophys. Res. Lett., 29, doi:10.1029/2002GL014903.

Fröhlich, C., and J. Lean, The sun's total irradiance: Cycles, trends, and related climate change uncertainties since 1976, Geophys. Res. Lett., 25, 4377-4380, 1998.

Gleissberg, W.: (1958), the 80-year sunspot cycle, Journal of Britih Astronomy Association 68, pg 150

Hoyt, D.V. and Schatten, K.H. (1997): The role of the sun in climate change, New York Oxford, Oxford University Press, 1997 
Hoyt, D.V. (1979): Variations in sunspot structure and climate, Climate Change, 2, pp 79-92

Labitzke, K., The global signal of the 11-year sunspot cycle in the stratosphere:

Differences between solar maxima and minima, Meteorol. Zeitschift, 10, 83-90, 2001.

Landscheidt, T, (2000). Solar wind near earth, indicator if variations in global temperatures in Vazquez,M. and Schmiedere, E, ed.: The solar cycle and terestrial climate, European Space Agency, Special Publication 463, pp 497-500

Palle Bago, E., and Butler, C.J. (2000): The influence of cosmic rays on terrestrial clouds and global warming, Astron. Geophysics, 41, 4.18-4.22

Palle Bago, E., and Butler, C.J. (2001): Sunshine records from Ireland: Cloud factors and possible links to solar activity and cosmic rays, International Journal of Climatology 21, 709-729

Scafetta, N., West, B.J. of Duke University, (2006). Phenomenological Solar Signature in 400 years of Reconstructed Northern Hemisphere Temperature Record", GRL.

Shaviv, N.J., (2002). The Spiral Structure of the Milky Way, Cosmic Rays, and Ice Age Epochs on Earth, New Astronomy, 8, pp 39-77

Shaviv, N.J. (2005). On climate response to changes in cosmic ray flux and radiative budget, Journal of Geophysical Research 110,

Shindell, D.T., D. Rind, N. Balachandran, J. Lean, and P. Lonergan, Solar cycle variability, ozone, and climate, Science, 284, 305-308, $1999 a$.

Soon, W.H., Posmentier, E., Baliunas, S.L. (1996), Inference of solar irradiance variability from terrestrial temperature changes 1880-1993: an astrophysical application of the sun-climate relationship. Astrophysical Journal 472, pp 891-902

Svenmark, H, Friis-Christensen, E.: (1997):Variation of cosmic ray flux and global cloud cover- a missing link in solar -climate relationships, Journal of Atmospheric and SolarTerrestrial Physics, 59, pp 1125-32

Theijl, P. and Lassen, K. (2000): Solar forcing of the northern hemisphere land air temperature, Journal Atmospheric Solar Terrestrial Physics, 62, pp 1207-1213

Tinsley, B.A., and Yu, F. (2002): Atmospheric ionization and clouds as links between solar activity and climate. In AGU monograph Solar Variability and its effects on the earth's atmosphere and climate system.

Veizer, J., Godderis, Y.,François, L.M. (2000). Evidence for decoupling of atmospheric CO2 and global climate during the Phanerozoic eon. Nature, 408 
Willson, $R$ (1997) Total solar irradiance trend during solar cycles 21 and 22: Science 277, pp 1963-1965

Willson, Richard C. and Mordvinov, Alexander V., 2003. Secular total solar irradiance trend during solar cycles 21-23. GRL Vol. 30, No 5, 1199, 